\title{
MALÁRIA NO ESTADO DE SÃO PAULO, BRASIL, 1980 a 1983*
}

\author{
Dalva Marly Valério Wanderley** \\ José Carlos Rehder de Andrade** \\ Luiz Carlos Meneguetti** \\ Maria José Chinelatto** \\ Araripe Pacheco Dutra**
}

\begin{abstract}
WANDERLEY, D.M.V. et al. Malária no Estado de São Paulo, Brasil, 1980 a 1983. Rev. Saúde públ., S. Paulo, 19: 28-36, 1985.

RESUMO: São analisados dados referentes à malária no Estado de São Paulo, no período de 1980 a 1983 . Foi verificado que $97,0 \%$ dos casos diagnosticados se originaram em outras áreas do país, e destes, 93,5\% da Região Amazônica que tem sofrido a implantação de projetos de desenvolvimento econômico, responsáveis por acentuados deslocamentos populacionais. Selecionou-se algumas características desta população que retorna das áreas de transmissão, relacionando-as com o motivo do deslocamento para a área endêmica e o local de diagnós. tico no Estado de São Paulo. Foram analisados os focos de malária ocorridos no período, vinculando-os com a introdução de casos importados ou a existência de áreas de malária residual. O resultado desta análise contribui para a orientação da vigilância epidemiológica, fornecendo subsídios para o controle da endemia.
\end{abstract}

UNITERMOS: Malária. Epidemiologia.

\section{INTRODUÇÃO}

O Programa de Erradicação da Malária no Estado de São Paulo vem sendo executado pela Secretaria de Estado da Saúde, desde 1959 , segundo normas técnicas internacionais adotadas pelo programa nacional ${ }^{9}$. Em sua fase preliminar, foi realizada a avaliação epidemiológica do Estado, que classificou os municípios em áreas de transmissão e não-maláricas. A partir desta fase de diagnóstico, teve seqüência o trabalho que previa ciclos semestrais de rociado intradomiciliar com DDT, associado ao diagnóstico e tratamento dos casos confirmados microscopicamente. Como conseqüência das atividades desenvolvidas, as áreas tiveram alterado seu quadro epidemiológico e, em 1964, começaram a ser transferidos os primeiros municípios para fa. ses mais avançadas do programa, o que significava medidas de vigilância diferenciadas em relação à situação anterior ${ }^{10}$.

Atualmente, o programa de erradicação tem o objetivo precípuo de manter a vigilância epidemiológica, no sentido de serem detectados precocemente casos de malária

* Trabalho apresentado na 36a Reunião Anual da Sociedade Brasileira para o Progresso da Ciência, São Paulo, julho de 1984.

* Da Superintendência de Controle de Endemias (SUCEN) da Secretaria de Estado da Saúde - Rua Tamandaré, 649 - 01525 - São Paulo, SP -- Brasil. 
WANDERLEY, D.M.V. et al. Malária no Estado de São Paulo, Brasil, 1980 a 1983. Rev. Sáide públ., S. Paulo, 19:28-36, 1985.

oriundos de áreas do país, e de outros países, onde a transmissão ainda não foi interrompida, assim como debelar os possíveis focos de transmissão que venham se instalar no Estado.

Nos últimos nove anos o número de casos de malária diagnosticados no Estado manteve-se praticamente constante, em média 1.194, com exceção de 1983 quando foram detectados 1.666 casos. O aumento de $31,3 \%$ da notificação no último ano, em rela. ção ao ano anterior (1.269), constituiu um reflexo da situação da endemia no paŕs. Dados da Superintendência de Campanhas de Saúde Pública (SUCAM) ${ }^{3}$ mostraram o registro de 264.523 novos casos no Brasil. Comparado com o ano anterior, este núme. ro representa um aumento de $19,2 \%^{5}$, e mantém a tendência crescente nos últimos anos. Vale assinalar que nem todos os casos ocorridos foram registrados. Estudos dos casos registrados no Brasil ${ }^{11}$, nos anos de 1981 e 1982 , mostraram que $99,3 \%$ e $99,7 \%$, respectivamente, ocorreram na Amazônia Legal. Esta situação repercute no Estado de São Paulo, onde 93,5\% dos casos diagnosti. cados procederam dessa Região.

Constituem objetivos, do presente traba. lho, a distribuição dos casos de malária, no período de 1980 a 1983, a análise de algumas características da população que retorna das áreas de transmissão, através do estudo das fichas de investigação epidemiológica de 1983, relacionando-as com o motivo do deslocamento destes indivíduos à área endêmica e o local de diagnóstico no Estado de São Paulo; e a apresentação dos focos esporádi. cos ocorridos no período e o ensaio de algumas explicações que possibilitem a compre. ensão da situação atual da endemia no Estado.

\section{MATERIAL E METTODOS}

Os procedimentos adotados pela Superintendência de Controle de Endemias (SUCEN), para a vigilância epidemiológica dos casos de malária estão a seguir apresentados.

Após a confirmação do caso de malária através do exame laboratorial, inicia-se a investigação epidemiológica do mesmo, através de formulário próprio. Vale ressaltar que cáso de malária deva ser entendido como a confirmação, pela observação microscópica, da presença de parasitos no sangue, existin. do ou não sintomas clínicos, ou seja, estão incluídos os portadores.

A ficha de investigação, numerada e identificada, contém o local (ou locais) de residência do doente (município, localidade), a espécie e densidade parasitária observadas, a movimentação do paciente e outros fatos que contribuam para elucidar a classificação do caso e determinar quando, e se possível, onde o indivíduo contraiu a infecção.

Os casos diagnosticados são classificados segundo recomendações da $\mathrm{OMS}^{6}$, nas seguintes categorias: a) importado, quando a infecção foi contrafda fora do Estado de São Paulo; b) recaída, quando corresponde ao reaparecimento de uma infecção anterior, por Plasmodium vivax, após ter sido completado o esquema de tratamento e a negativação da parasitemia sanguínea, sem deslocamento do paciente para áreas endêmicas; c) induzido, quando relàcionado com uma transfusão sanguínea ou outra forma de inoculação parenteral, mas não com a transmissão natural pelo mosquito; d) introduzido, quando derivado diretamente de um caso importado conhecido, em áreas onde a transmissão foi interrompida; e) autóctone, quando o caso se origina em localidade do Estado de São Paulo, existindo aí a fonte de infecção. Quando o caso não puder ser classificado nas categorias citadas, o mesmo é considerado autóctone, como forma de aprimorar a qualidade da vigilância.

A uma localidade com um ou mais casos autóctones, presença de vetores e condições ambientais favoráveis à transmissão, dá-se o nome de "foco de malária"? Neste caso, uma investigação epidemiológica mais detalhada é realizada, incluindo uma descrição da área, um histórico das medidas de controle adotadas na localidade (busca de casos, rociados), resultados das pesquisas entomológicas executadas por ocasião da transmissão atual, com o objetivo de confirmar a autoctonia.

Após a análise e classificação dos casos 
WANDERLEY, D.M.V. et al. Malária no Estado de São Paulo, Brasil, 1980 a 1983. Rev. Saride públ., S. Paulo, 19:28-36, 1985.

pelos diversos Serviços Regionais da SUCEN, todas as investigaçoes são encaminhadas ao nivel central, área de Epidemiologia, onde são revistas e agrupadas por local de transmissão (Estado, município e localidade) e as informações obtidas são enviadas à SUCAM e ao Centro de Informações de Saúde (CIS) da Secretaria de Estado da Saúde.

Foram objeto de estudo 5.370 casos de malária diagnosticados, investigados e classificados no período de 1980 a 1983, no Estado de São Paulo.

Com a finalidade de caracterizar a relação entre o deslocamento de grupos populacionais e a introdução de casos de malária no Estado de São Paulo, foi realizado um estudo das fichas de investigação epidemioló. gica dos 1.261 casos classificados como importados, nó ano de 1983. Adotou-se como critério a seleção dos casos residentes no Estado de São Paulo que se deslocaram para atuar em projetos de mineração, em projetos agro-pecuários, em construção de hidrelétricas, em atividades de transporte de cargas e aqueles em busca de lazer. Foi possível recuperar 1.012 fichas de investigação $(62,4 \%)$. A perda de $37,6 \%$ foi atribuída às falhas relacionadas ao preenchimento e ao tipo de informação solicitada nas fichas, além do fato de os doentes năo indicarem residência no Estado de Sáo Paulo.

Os casos autóctones foram estudados através dos relatórios de focos.

\section{RESULTADOS E DISCUSSÃO}

A classificação dos casos analisados no periodo, por espécie de plasmódio diagnosticada, encontra-se na Tabela 1. Fica eviden. te o predomínio de malária vivax entre os casos detectados. Entretanto, a percentagem de Plasmodium falciparum $(25,7 \%)$ não teve distribuição homogênea ao longo do período, de 1980 a 1982 foi de $23,7 \%$ e em 1983 de $30,2 \%$. Uma das prováveis causas deste aumento é a existência de cepas de $P$. falciparum resistentes aos medicamentos comumente utilizados, tais como 4-aminoquinoleínas ${ }^{11}$ e sulfa-pirimetamina ${ }^{2}$, representando, do ponto de vista epidemiológico, maior perfodo de parasitemia circulante nos pacientes $e$, conseqüentemente, maior possibi. lidade de infecção de anofelinos, com elevação do nivel de transmissão.

T A B E L A 1

Casos de malária investigados, segundo espécie de plasmódio. Estado de São Paulo, 1980 a 1983.

\begin{tabular}{|c|c|c|c|c|c|}
\hline \multirow{2}{*}{$\begin{array}{l}\text { Classificaçāo } \\
\text { dos casos }\end{array}$} & \multicolumn{3}{|c|}{ Espécie de plasmódio } & \multicolumn{2}{|c|}{ Total } \\
\hline & $\bar{P}$. vivax & P. falciparum & $\overline{\text { Associado }}$ & no & $\%$ \\
\hline $\begin{array}{l}\text { Autóctones } \\
\text { Introduzidos } \\
\text { Importados } \\
\text { Induzidos } \\
\text { Recaídas }\end{array}$ & $\begin{array}{r}114 \\
8 \\
3604 \\
15 \\
10\end{array}$ & $\begin{array}{c}11 \\
- \\
1370 \\
- \\
-\end{array}$ & $\begin{array}{c}\overline{1} \\
237 \\
-\end{array}$ & $\begin{array}{r}126 \\
8 \\
5211 \\
15 \\
10\end{array}$ & $\begin{array}{r}2,35 \\
0,15 \\
97,04 \\
0,28 \\
0,19\end{array}$ \\
\hline $\begin{array}{l}\text { Total } \\
\text { (\%) }\end{array}$ & $\begin{array}{c}3751 \\
(69,85)\end{array}$ & $\begin{array}{c}1381 \\
(25,72)\end{array}$ & $\begin{array}{c}238 \\
(4,43)\end{array}$ & $\begin{array}{c}5370 \\
(100,00)\end{array}$ & 100,00 \\
\hline
\end{tabular}

Fonte: SUCEN 
WANDERLEY, D.M.V. et al. Malária no Estado de São Paulo, Brasil, 1980 a 1983. Rev. Sauide ptibl., S. Paulo, 19: 28-36, 1985.

Marques e Pinheiro ${ }^{4}$ chamam a atenção para os deslocamentos dos casos de malária da Amazônia Legal para outras regioes brasi. leiras, reintroduzindo a endemia em áreas onde já havia sido interrompida a sua transmissão. O Estado de Sáo Paulo encabeça a relação dos Estados para onde convergiram as principais correntes de indivíduos maláricos. Dos 5.211 casos classificados como importados, no peŕodo de 1980 a $1983,96,3 \%$ procederam da Amazônia Legal. A Tabela 2 mostra que $85,9 \%$ dos casos foram procedentes de apenas três Estados da Federação: Rondônia, Mato Grosso e Pará. O percentual maior de casos procedentes de Rondônia e Mato Grosso coincide com a chamada "penetração sulista" na Amazônia, através do Centro-Oeste, em direção a estes Estados ${ }^{1}$.

O estudo realizado em 1.012 investigaçőes epidemiológicas de casos importados, em 1983, permitiu verificar que $49,6 \%$ esta- vam relacionados com projetos de minera. ção, o que foi constatado nas várias regiðes do Estado, com exceção de Sáo José do Rio Preto e Presidente Prudente, cuja relação maior foi com a atividade de transporte. Esta atividade foi responsável por $29,0 \%$ dos casos do Estado. Os projetos agro-pecuários contribuíram com $14,1 \%$, embora a região de Campinas tenha apresentado $44,2 \%$ dos doentes relacionados com esta atividade. Este percentual parece ser justificado pela existência na região de uma empresa recrutadora de mão-de-obra para tal tipo de atividade no Estado de Mato Grosso, município de Colíder, de onde procederam 77,5\% dos 89 casos importados deste Estado, diagnosti. cados na área. A sarda de pessoas, em busca de lazer, totalizou $6,5 \%$ dos casos em estudo, chamando à atenção os $23,8 \%$ obtidos na região de Ribeirão Preto. A construção de hidrelétricas e ferrovia contribufram com,

T A B E L A 2

Procedência dos casos importados de malária no Estado de Sŕo Paulo, 1980 a 1983.

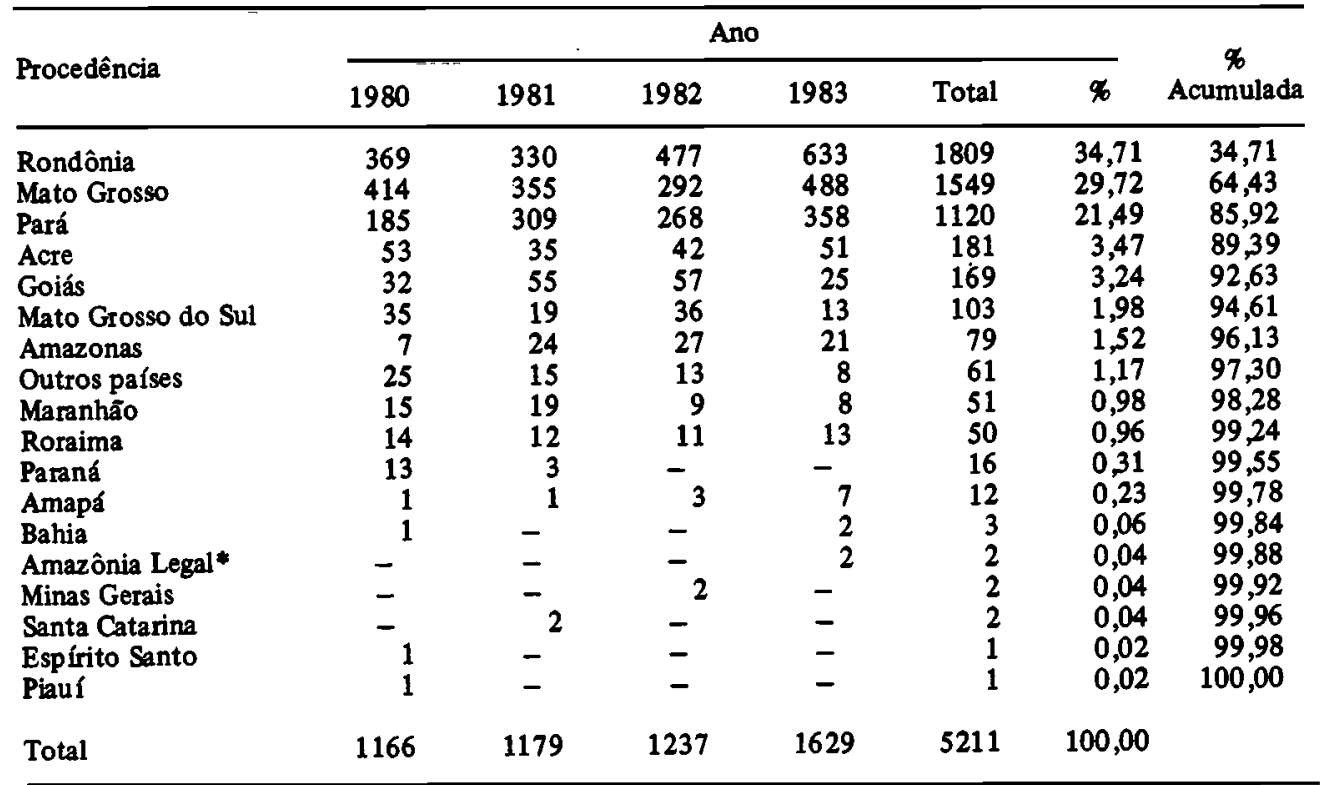

Fonte: SUCEN

* Nâo foi possível determinar o Estado. 
WANDERLEY, D.M.V. et al. Malária no Estado de São Paulo, Brasil, 1980 a 1983. Rev. Saúde pribl, S. Paulo, 19: 28-36, 1985.

respectivamente, $0,7 \%$ e $0,1 \%$ dos casos.

Os municípios da Amazônia Legal mais comprometidos com os casos de malária atendidos no Estado de São Paulo foram: do Estado de Rondônia - Porto Velho $(60,2 \%)$ e Ariquemes $(20,4 \%)$; do Estado de Mato Grosso - Colíder $(51,8 \%)$ e Alta Floresta $(18,2 \%)$. Com relação ao Estado do Pará, 60,8\% dos casos procederam dos municípios de Itaituba, Conceição do Araguaia e Marabá.

A distribuição dos casos investigados, em 1983 , por sexo, mostrou predomínio do sexo masculino $(87,4 \%)$. Quanto ao grupo etário, as faixas de 20 a 29 anos $(35,6 \%)$ e de 30 a 39 anos $(29,3 \%)$ representaram $64,9 \%$ dos casos. Esta participação maior de indivíduos jovens e do sexo masculino sugere 0 deslocamento de mão-de-obra em direção às fronteiras da Amazônia Legal, representada por indivíduos que vão em busca de emprego, por recrutados pelas empresas que atuam na área e por aqueles cujo trabalho exige constante movimentação para a região. A mobilidade de grupos familiares relacionada com atividades de colonização ${ }^{12}$, que pode ser representada pelos casos de malária em menores de 10 anos e em elementos do sexo feminino, mostrou-se menor do que a verificada em levantamentos realizados no Estado do Rio Grande do Sul, que revelaram uma participação maior de mulheres ${ }^{3}$. Baseando-se em Prothero ${ }^{8}$, a mobilidade dos portado. res de malária, atendidos no Estado de São Paulo, pode ser definida como circulação, ou seja, deslocamento para fora do lugar de residência, mas com eventual retorno. Vale ressaltar que o número de casos de malária importados no Estado de São Paulo apresentou um aumento no final de 1983, coincidindo com as comemorações da época, desativação de garimpos e período de chuvas intensas, o que poderia justificar o retorno da mão-de-obra que se dirigiu à Amazônia.

Tendo em vista que a penetração de indi. víduos infectados em áreas receptivas, isto é, com presença de mosquitos vetores, pode dar início a transmissão, merece destaque ainda com relação aos casos importados, a distribuição dos mesmos por Serviço Regio- nal da SUCEN, responsável pelo diagnóstico. Nota-se na Tabela 3 que a Grande São Paulo, ou mais precisamente o laboratório central da SUCEN, foi responsável pelo atendimento de $35,1 \%$ dos casos detectados no Estado, no período de 1980 a 1983 . A concentração de sedes de empresas responsáveis pelo desenvolvimento de projetos em áreas de transmissão, que aqui recrutam pessoal para trabalhar e, ainda o fato de a Capital constituir-se em polo de atração como possibilidade de emprego, além de melhores condições de assistência médica, poderiam fazer parte da explicação do elevado percentual de casos diagnosticados na Grande São Paulo.

Dos 134 casos classificados como autóctones e introduzidos (Tabela 4) no período de 1980 a 1983 merecem destaque os de infecção por $P$. falciparum, em virtude da gra. vidade do quadro clínico. No ano de 1981 ocorreu um foco com características de surto epidêmico na regiáo de São José do Rio Preto, municípios de Marinópolis e Palmeira D'Oeste. Estudos realizados na ocasião caracterizaram a receptividade da área, através da presença de Anopheles $(N)$ darlingi e a vulnerabilidade representada por pescadores amadores e profissionais, provenientes de vários pontos do país, que freqüentavam as margens do Rio São José dos Dourados. De fato, foi em conseqüència da permanência na regiāo de dois casos importados do Esta. do do Mato Grosso, que apresentaram recrudescências, em datas coincidentes com os períodos de transmissáo, que nove casos aconteceram. Um dos casos permaneceu 12 dias sem diagnóstico por parte dos Serviços de Saúde da área, evoluindo para óbito. Alertados para a situação, medidas para o controle e seguimento do foco foram adotadas e mostraram-se eficazes.

Os outros dois casos registrados no periodo, com diagnóstico de malária falciparum, ocorreram nos municípios de Miracatu (em 1980) e Campinas (em 1983). O caso classificado como autóctone de Miracatu, Divisão Especial do Vale do Ribeira (DEVALE), não foi objeto de investigação exaustiva, permanecendo a dúvida com relação a presença 
WANDERLEY, D.M.V. et al. Malária no Estado de São Paulo, Brasil, 1980 a 1983. Rev. Saúde pribl., S. Paulo, 19: 28-36, 1985.

T A B E L A 3

Distribuição dos casos importados de malária no Estado de São Paulo, 1980 a 1983.

\begin{tabular}{|c|c|c|c|c|c|c|c|}
\hline \multirow{2}{*}{$\begin{array}{l}\text { Serviço Regional } \\
\text { da SUCEN }\end{array}$} & \multicolumn{6}{|c|}{ Ano } & \multirow{2}{*}{$\begin{array}{c}\% \\
\text { Acumulada }\end{array}$} \\
\hline & 1980 & 1981 & 1982 & 1983 & Total & $\%$ & \\
\hline Grande São Paulo & 392 & 429 & 433 & 574 & 1828 & 35,08 & 35,08 \\
\hline Presidente Prudente & 201 & 137 & 185 & 205 & 728 & 13,97 & 49,05 \\
\hline Campinas & 116 & 134 & 125 & 193 & 568 & 10,90 & 59,95 \\
\hline São José R. Preto & 116 & 114 & 132 & 170 & 532 & 10,21 & 70,16 \\
\hline Ribeirão Preto & 99 & 122 & 120 & 131 & 472 & 9,06 & 79,22 \\
\hline Marflia* & 90 & 83 & 91 & 158 & 422 & 8,10 & 87,32 \\
\hline Araçatuba & 90 & 79 & 54 & 72 & 295 & 5,66 & 92,98 \\
\hline Sorocaba & 31 & 40 & 47 & $4 \overline{7}$ & 165 & 3,17 & 96,15 \\
\hline São Vicente** & 27 & 32 & 28 & 51 & 138 & 2,65 & 98,80 \\
\hline Taubaté & 4 & 9 & 22 & 28 & 63 & 1,20 & 100,00 \\
\hline Total & 1166 & 1179 & 1237 & 1629 & 5211 & 100,00 & \\
\hline
\end{tabular}

Fonte: SUCEN

* inclui os municípios da Região Administrativa de Bauru

* inclui os municípios do DEVALE

de fonte de infecção importada na área, ou mesmo história de deslocamentos para outras regiões, não mencionadas na investigação. $O$ caso autóctone do município de Campinas é o primeiro registrado nos últimos 25 anos. Este município, no decorrer da elaboração do Plano de Erradicação da Malária no Estado de São Paulo ${ }^{9}$, não foi incluído como sendo área malárica, porque a transmissão já não existia. O foco restringiu-se a um caso, cujo paciente afirmou não ter saído do município, nos últimos dois anos. Distando $3 \mathrm{~km}$ do local de residência existem lagoas e açudes, por ele freqüentados e utilizados para pesca, que foram considerados como provável local da infecção. A pesquisa entomológica, realizada uma semana após a detecçāo do caso, indicou razoável densidade anofélica, com predomínio de Anopheles ( $N)$ albitarsis albitarsis $(80,4 \%)$, vetor secundário que pode ter sido responsável pela picada infectante.

Dos casos autóctones de infecção por $P$. vivax, 67,1\% ocorreram na Região do Lito. ral do Estado (Baixada Santista, Vale do Ribeira e Litoral Norte), atribuídos a uma transmissão local constante, com caracterís- ticas de malária residual, ou possivel introdução desconhecida. Estas áreas apresentam clima quente e úmido, o que propicia o aparecimento de plantas epífitas, sendo grande a quantidade de bromélias. Nestas condiçōes, a existência de espécies do subgênero Kerteszia tem sido comprovada nas constantes pesquisas entomológicas efetuadas $(A$. $(K$.) cruzii e $A$. (K.) bellator). A esta receptividade se associa uma vulnerabilidade dependente da procura da área por projetos de exploração vegetal/mineral, projetos de construção civil ou mesmo turismo. Alguns dos locais onde foram registrados casos autóctones sáo de difícil acesso à vigilância, necessitando melhor caracterização a fim de elucidar se constituem "áreas de malária residual", caracterizadas pela resposta do vetor às açס̄es de controle e pela presença de portadores assinto. máticos.

A partir de 1980 , têm sido registrados casos autóctones esporádicos no município de Juquitiba, único localizado na Regiao da Grande Sáo Paulo que foi considerado como originalmente de transmissão. A detecção de dois casos, em 1982, conduziu a realização da coleta de sangue, para reação de imuno- 
WANDERLEY, D.M.V. et al. Malária no Estado de São Paulo, Brasil, 1980 a 1983. Rev. Saúde públ., S. Paulo, 19:28-36, 1985.

T A B E L A 4

Casos autóctones e introduzidos de malária segundo regiâo e município. Estado de Sāo Paulo, 1980 a 1983.

\begin{tabular}{|c|c|c|c|c|c|c|}
\hline \multirow[b]{2}{*}{ Região } & \multirow[b]{2}{*}{ Município } & \multicolumn{4}{|c|}{ Ano } & \multirow[b]{2}{*}{ Total } \\
\hline & & 1980 & 1981 & 1982 & 1983 & \\
\hline Grande São Paulo & $\begin{array}{l}\text { Embu } \\
\text { Embu Guaçu } \\
\text { Itapecerica da Serra } \\
\text { Juquitiba }\end{array}$ & $\begin{array}{l}- \\
- \\
3\end{array}$ & $\begin{array}{l}- \\
\overline{1} \\
\overline{1}\end{array}$ & $\begin{array}{l}- \\
\overline{2}\end{array}$ & $\begin{array}{l}1 \\
1 \\
1 \\
1\end{array}$ & $\begin{array}{l}1 \\
1 \\
1 \\
7\end{array}$ \\
\hline Baixada Santista & $\begin{array}{l}\text { Guarujá } \\
\text { Itanhaém } \\
\text { Mongaguá } \\
\text { Praia Grande } \\
\text { Santos } \\
\text { São Vicente }\end{array}$ & $\begin{array}{l}\overline{3} \\
1^{*} \\
\overline{1}\end{array}$ & $\begin{array}{l}- \\
\overline{1} \\
\overline{2} \\
-\end{array}$ & $\begin{array}{l}\overline{2} \\
\overline{2} \\
\overline{2}\end{array}$ & $\begin{array}{l}1 \\
- \\
- \\
- \\
-\end{array}$ & $\begin{array}{l}1 \\
5 \\
2 \\
2 \\
2 \\
3\end{array}$ \\
\hline DEVALE & $\begin{array}{l}\text { Cananéia } \\
\text { Iguape } \\
\text { Iporanga } \\
\text { Juquiá } \\
\text { Miracatu } \\
\text { Pedro de Toledo } \\
\text { Peruibe } \\
\text { DEVALE Mun. Indet. }\end{array}$ & $\begin{array}{c}1 \\
2 \\
- \\
- \\
3 \\
\overline{31} \\
-\end{array}$ & $\begin{array}{l}1 \\
- \\
\overline{1} \\
- \\
- \\
\overline{1}\end{array}$ & $\begin{array}{l}\overline{1} \\
1 \\
- \\
- \\
\overline{4} \\
-\end{array}$ & $\begin{array}{l}\overline{2} \\
\overline{-} \\
\overline{2} \\
6 \\
-\end{array}$ & $\begin{array}{r}2 \\
5 \\
1 \\
1 \\
3 \\
2 \\
41 \\
1\end{array}$ \\
\hline Litoral Norte & $\begin{array}{l}\text { Caraguatatuba } \\
\text { Sâo Sebastião } \\
\text { Ubatuba }\end{array}$ & $\begin{array}{l}1 \\
4 \\
2\end{array}$ & $\begin{array}{r}1 \\
1 \\
-\end{array}$ & $\begin{array}{r}1 \\
3 \\
-\end{array}$ & $\begin{array}{l}4 \\
1 \\
3\end{array}$ & $\begin{array}{l}7 \\
9 \\
5\end{array}$ \\
\hline Sorocaba & Tapiraí & - & 1 & - & - & 1 \\
\hline Campinas & Campinas & - & - & - & 1 & 1 \\
\hline Bauru & Lins & 1 & - & - & - & 1 \\
\hline São José R. Preto & $\begin{array}{l}\text { Icém } \\
\text { Marinópolis } \\
\text { Palmeira D'Oeste } \\
\text { Tabapuã }\end{array}$ & $\begin{array}{l}- \\
- \\
-\end{array}$ & $\begin{array}{r}2 \\
4 \\
5 \\
-\end{array}$ & $\begin{array}{l}- \\
\overline{-} \\
-\end{array}$ & $\begin{array}{l}- \\
- \\
-\end{array}$ & $\begin{array}{l}2 \\
4 \\
5 \\
1\end{array}$ \\
\hline Araçatuba & Castilho & - & - & - & 3 & 3 \\
\hline Presidente Prudente & $\begin{array}{l}\text { Panorama } \\
\text { Presidente Epitácio } \\
\text { Teodoro Sampaio }\end{array}$ & $\begin{array}{l}- \\
-\end{array}$ & $\bar{z}$ & $\frac{3^{* *}}{6^{* *}}$ & $\bar{z}$ & $\begin{array}{l}3 \\
4 \\
6\end{array}$ \\
\hline Marîlia & São Pedro do Turvo & - & $1 *$ & - & - & 1 \\
\hline & Total & 57 & 22 & 27 & 28 & 134 \\
\hline
\end{tabular}

Fonte: SUCEN

- 1 caso introduzido

* 2 casos introduzidos 
WANDERLEY, D.MV. et al. Malária no Estado de São Paulo, Brasil, 1980 a 1983. Rev. Saúde públ., S. Paulo, 19: 28-36, 1985.

fluorescência indireta para o diagnóstico da infecção malárica, em 736 moradores da localidade, resultando 58 positivos $(7,9 \%)$ para anticorpos da classe IgG, confirmando antecedentes da endemia na população. Ape. sar de não ter sido feito pesquisa entomoló. gica na oportunidade, em pesquisas anteriores foi demonstrada a presença de $A$. $(K$. cruzii e $A$. (K.) bellator. 0 munić́pio de Juquitiba, assim como os demais onde ocorreram casos em 1983, apresentam contigüidade com a Região do Vale do Ribeira, asso. ciado ao fato de estarem ligados pela Rodovia Régis Bittencourt (BR-116). Estes casos podem ser relacionados também, com a possível introdução desconhecida em áreas com presença de anofelinos do subgênero Kerteszia, ou focos residuais de malária.

Com relação aos focos de mialária ocorridos em municípios fronteiriços aos Rios $\mathrm{Pa}$ raná e Grande, a situação epidemiológica difere dos anteriormente citados. Nesses locais, a movimentaç̃̃o de grupos populacionais é intensa devido a existência de rodovias que se comunicam com Estados do Centro. -Oeste do País, além dos projetos de implan. tação de usinas e do comércio de pescado na região. A esta vulnerabilidade associa-se a presença do Anopheles ( $N$ ) darlingi, principal vetor responsável pela transmissáo da malária, e de espécies secundárias, das quais se desconhece a importância epidemiológica. Nessas áreas, a presença de fonte de infeç̧ão importada, nem sempre identificada, pode desencadear focos de malária, que exigem, por parte da SUCEN, rapidez na localização dos casos, na sua investigação epidemiologica e no seu tratamento, além das medidas de ro. ciado com DDT.

Em virtude da diminuição da transmissáo da malária, ao longo dos anos, no Estado de Săo Paulo, a preocupação com a endemia deixou de ser uma realidade para a popula. ção e a categoria médica. Tal fato pode ter contribuído para o registro, em 1983, nas fichas de investigação epidemiológica, de oito óbitos por malária, decorrentes do diagnosti co tardio ou conduta terapêutica inadequada. Todos os casos eram importados e tratavam-se de individuos do sexo masculino, $87,5 \%$ com idades superiores a 40 anos e estavam internados quando da ocorrencia do óbito, e à SUCEN náo foi possível precisar a conduta terapêtica instituída. Todos os 6bitos foram por malária falciparum, sendo que em dois a malária vivax estava associada. Diante desse quadro, a SUCEN reativou a divulgação da malária junto à população e aos médicos, com o objetivo de motivar a participaçăo, visando o diagnóstico precoce e correto dos casos e a observância das normas terapêuticas preconizadas.

Um dos fatores fundamentais para a fase de controle em que se encontra o Estado de São Paulo, visando o incremento da vigilân. cia epidemiológica, é a necessidade da participação da rede básica de saúde, objetivando diagnóstico mais precoce, evitando-se o esta. belecimento de focos e o aparecimento de formas graves.

\section{CONCLUSÓES}

A mobilidade de doentes de malária, oriundos principalmente da Região Amazônica, pode reintroduzir a endemia em áreas do Estado de São Paulo.

Os casos de transmissão local, em áreas com presença de subgênero Kerteszia, neces. sitam melhor avaliação, a fim de comprovar a existência de focos residuais.

Tendo em vista que as medidas de controle utilizadas, até o presente momento, não têm sido suficientes para controlar a expan. săo da endemia na Amazônia Legal, expondo o Estado de São Paulo a possível reintrodução da mesma, faz-se necessário a incorporação do estudo da relação do social com o biológico, com o objetivo de aprofundar a análise e propiciar melhor direcionamento da vigilância epidemiológica. 
WANDERLEY, D.M.V. et al. Malária no Estado de São Paulo, Brasil, 1980 a 1983. Rev. Saúde públ., S. Paulo, 19: 28-36, 1985.

WANDERLEY, D.M.V. et al. [Malaria in S. Paulo State, Brazil, 1980 up to 1983]. Rev. Saúde públ., S. Paulo, 19: 28-36, 1985.

ABSTRACT: Data on malaria in S. Paulo State, Brazil, in the period from 1980 to 1983 are analysed. It was found that $97.0 \%$ of the cases diagnosed had their origin in other areas of the Country. Of this percentage, $93.5 \%$ came from the Amazonian region where the projects for economic development responsible for increased population displacement have been established. Some features of this population that retums to areas of transmission were selected and relating to the reason for the migration into the endemic area and the site of diagnosis in S. Paulo State. The malarial focuses which occurred during the period mentioned were analysed and correlated with the introduction of the immigrant cases or the existence of reșidual malaria areas. The results of this analysis contributes to the efectiveness of the control of epidemiological surveillance and it provides data for the control of endemic malaria.

UNITERMS: Malaria. Epidemiology.

\section{REFEREENCIAS BIBLIOGRÁFICAS}

1. ALMEIDA, A.L.O. de A expansão da fronteira. Ciênc. Hoje, 10(2): 38-9, 1984.

2. BOULOS, M. \& BARATA, L.C.B. Avaliação do esquema sulfapirimetamina para malária falciparum. In: Congresso da Sociedade Brasileira de Medicina Tropical, 20\%, Salvador, 1984. Salvador, 1984. p. 43.

3. MALARIA no Rio Grande do Sul: 1976-1982. IES - Inf. epidemiol SUCAM, 2(14), 1983.

4. MARQUES, A.C. PINHEIRO, E.A. Fluxos de casos de malária no Brasil em 1980. Rev. bres. Malar., 34: 1-31, 1982.

5. MINISTERIO DA SAODE. Superintendência de Campanhas de Saúde Pública. Relatoro anual Brasília, 1982.

6. ORGANIZAÇĀO MUNDIAL DA SAÓDE. Classificagēo dos casos de malaria. Genebra, 1961. (WHO/PA/164.61).

7. PAMPANA, E. Erradicacion de la malaria. México, Centro Regional de Ayuda Técnica, 1963.
8. PROTHERO, R.M. Doença e mobilidade: uma questão negligenciada em epidemiologia. In: Seminário sobre Transmissão e Controle de Doenças Tropicais no Processo de Migração Humana, Bras lila, 1982. Anais. Brastlia, 1982. p. 169-81.

9. SECRETARIA DA SAODE. Serviço de Ptofilaxia da Malária. Plano pare a erradicafāo da malaria no Estado de São Paulo, Brasil. Sấo Paulo, 1958.

10. SECRETARIA DA SAODE. Superintendência de Controle de Endemias - SUCEN. Relatório sobre avaliagio epidemiologica da campanha de erradicaçato da maldria no Es. tado de São Paulo (feveretro de 1960 a setembro de 1963). São Paulo, 1963.

11. TAUIL, P.L. Malária: agrava-se o quadro da doença no Brasil. Ciénc. Hoje, 11(2):5864,1984 .

12. VELHO, O. Por que se migra na Amazônia. Ciênc. Hoje, 10(2): 34-9, 1984.

Recebido para publicação em 23/08/1984. Aprovado para publicagīo em 17/12/1984. 